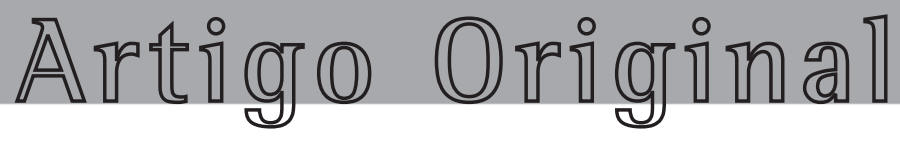

\title{
Prevalência de asma em funcionários de hospital universitário avaliada por meio de questionário de saúde respiratória da Comunidade Européia *
}

\author{
Asthma prevalence among employees of a university hospital as evaluated using a \\ European Union respiratory health questionnaire
}

\author{
ANTÔNIO SOARES AGUIAR FILHO', EDMUNDO PESSOA ALMEIDA LOPES NETO², EMANUEL SÁVIO CAVALCANTI SARINHO², \\ MARIA MAGALHÃES VASCONCELOS ${ }^{4}$, DARLA SIQUEIRA TENÓRIO LIMA ${ }^{4}$, PENÉLOPE MATOS WIRTSBIKI ${ }^{4}$
}

\begin{abstract}
RESUMO
Objetivo: Determinar a prevalência de asma brônquica em funcionários de um hospital universitário, com idades entre 20 e 44 anos, segundo os critérios do European Community Respiratory Health Survey. Métodos: Através de estudo descritivo, prospectivo, aleatório e estratificado de prevalência, foram analisados os questionários de 351 funcionários do Hospital das Clínicas da Universidade Federal de Pernambuco, entre abril e outubro de 2002. Foram consideradas as variáveis de caracterização amostral, as independentes referentes a sinais e sintomas e a dependente (asma, caracterizada pela informação de crise, diagnosticada por médico, nos doze meses anteriores à pesquisa). Resultados: A prevalência de asma foi de 10,7\%. Dispnéia noturna, sensação de aperto torácico e tosse noturna foram os sintomas mais freqüentes, com significância só no sexo feminino (respectivamente $p=0,03, p=0,04$ e $p=0,001$ ). Dispnéia noturna, sibilos nos últimos doze meses, dispnéia com sibilos e uso de medicação para asma predominaram entre 20 e 29 anos, com significância para os dois últimos sintomas $(\mathrm{p}<0,001)$. Houve 35 casos de subtratamento de asma $(10,7 \%)$. Conclusão: Este estudo, pioneiramente analisando casuística de funcionários de um hospital universitário de país em desenvolvimento, mostra que a prevalência de asma foi um pouco mais elevada que a encontrada na literatura e sugere que o trabalho em hospital universitário não reduz a freqüência de subtratamento.
\end{abstract}

Descritores: Asma/diagnóstico; Asma/epidemiologia; Educação em saúde; Hospitais universitários; Questionários

\begin{abstract}
Objective: To determine the prevalence of bronchial asthma among university hospital employees between the ages of 20 and 44 based on the criteria established in the European Community Respiratory Health Survey. Methods: A descriptive, prospective, randomized, stratified prevalence study was conducted, in which questionnaires completed between April and October of 2002 by 351 employees of the Universidade Federal de Pernambuco (Federal University at Pernambuco) Hospital das Clínicas were evaluated. For sample characterization, the independent variables used were the signs and symptoms of asthma, and one dependent variable (physician-diagnosed asthma, characterized by data related to attacks occurring within the 12 months preceding the study) was included. Results: The prevalence of asthma was 10.7\%. Nocturnal dyspnea, chest tightness and nocturnal cough were the most frequent symptoms, although statistically significant correlations with asthma were found exclusively among females $(p=0.03, p=0.04$ and $p=0.001$, respectively). Nocturnal dyspnea, wheezing within the last 12 months, dyspnea with wheezing and the use of asthma medication were more frequent among individuals between 20 and 29 years of age. The last two variables presented statistical significance $(p<0.001)$. Undertreatment of asthma was identified in 35 (10.7\%) of the cases. Conclusions: The results of this pioneering study show that asthma prevalence in a sample composed of employees of a university hospital located in a developing country was slightly higher than that found in the literature and suggest that working in a university hospital does not reduce the frequency of undertreatment.
\end{abstract}

Keywords: Asthma/diagnosis; Asthma/epidemiology; Hospitals, university; Questionnnaires

\footnotetext{
* Trabalho realizado no Hospital das Clínicas da Universidade Federal de Pernambuco - UFP - Recife (PE) Brasil.

1. Mestre em Medicina Interna, Professor Assistente da Universidade Federal de Pernambuco - UFP - Recife (PE) Brasil.

2. Professor Doutor em Gastroenterologia, Hospital das Clínicas da Universidade Federal de Pernambuco - UFP - Recife (PE) Brasil.

3. Professor Doutor em Pediatria, Hospital das Clínicas da Universidade Federal de Pernambuco - UFP - Recife (PE) Brasil.

4. Acadêmicos de Medicina da Universidade Federal de Pernambuco - UFP - Recife (PE) Brasil.

Endereço para correspondência: Antônio Soares Aguiar Filho. Rua Dona Benvinda 258 - CEP 50070-220, Boa Vista, Recife (PE) Brasil.

Tel.: 5581 3423-0628. E-mail: aaguiar@hotlink.com.br

Recebido para publicação em 8/6/04. Aprovado, após revisão, em 16/5/05.
} 


\section{INTRODUÇÃO}

0 aspecto mais intrigante da epidemiologia descritiva da asma e dos sintomas asma-símile é sua variabilidade geográfica e temporal. Diversos indicadores epidemiológicos, tais como taxas de morbidade e mortalidade, internações hospitalares e quantidades de drogas prescritas, sugerem que a prevalência da asma tem aumentado significativamente desde $1970 . .^{(1-2)}$

Esse fenômeno é particularmente evidente nas áreas urbanizadas de países em desenvolvimento, embora o aumento da morbidade da asma tenha sido registrado também em países desenvolvidos. ${ }^{(3-4)}$

Foi essa problemática que desencadeou o esforço internacional para a elaboração de questionários, objetivando a padronização dos levantamentos epidemiológicos, especialmente quanto ao conceito de asma. Para a faixa etária de 20 a 44 anos foi desenvolvido o questionário European Community Respiratory Health Survey (ECRHS).

0 Brasil é um país carente de dados epidemiológicos fidedignos, fato que dificulta o planejamento e a execução de medidas preventivas e educacionais para os problemas relacionados à saúde, voltados para uma parcela expressiva da população. ${ }^{(5)}$ Os dados epidemiológicos do Sistema Único de Saú$\mathrm{de}^{(6)}$ são os mais abrangentes, no entanto, devem ser analisados com cautela, por conterem erros. ${ }^{(7)}$ Em várias regiões do país, os casos de asma não são notificados, enquanto que, em outras, essas informações são um artifício utilizado pelos hospitais para obter maior remuneração.

Mesmo considerando esse fato, as estatísticas são alarmantes. Em 1996, a asma foi a quarta maior causa de hospitalização no Brasil, correspondendo a cerca de 350.000 internações em todo o país, a um custo de aproximadamente 76 milhões de reais, o que equivaleu ao terceiro maior gasto do Sistema Único de Saúde com uma doença específica, segundo dados do 111 Consenso Brasileiro no Manejo da Asma da Sociedade Brasileira de Pneumologia e Tisiologia. Além disso, a asma foi responsável por uma média de 2.000 óbitos/ano, dos quais $70 \%$ ocorreram durante a hospitalização. ${ }^{(8)}$

Os custos diretos com o tratamento da asma correspondem à soma dos gastos com medicamentos, aparelhos, consultas médicas e hospitalizações. Os custos indiretos envolvem absenteísmo à escola e ao trabalho, tanto do paciente, quanto de seu acompanhante, além de perda de produtividade, aposentadoria precoce e morte. ${ }^{(9)}$

Em 1995, demonstrou-se que o aumento da morbidade e da mortalidade observadas na asma se associa, entre outros fatores, à inadequação da abordagem médica e à fraca adesão dos pacientes ao tratamento, o qual, por ser complexo, exige participação ativa de seus portadores e dos familiares. Pelo fato da asma levar a limitações físicas, emocionais e sociais, as condutas terapêuticas requerem, além do tratamento farmacológico adequado, que o doente tenha noções sobre a doença, fatores desencadeantes e forma de evitá-los, assim como adquira habilidades para o uso correto das medicações. ${ }^{(10)} A$ estas necessidades associa-se o reconhecimento dos sinais de controle e de descontrole da doença. ${ }^{(11)}$

Com base nessas premissas, qualquer programa de tratamento, educação ou prevenção para asma deve ser precedido e seguido por levantamentos epidemiológicos baseados em questionários padronizados, para o acompanhamento do processo, análise de efetividade dos programas e correções necessárias. ${ }^{(12)}$

As pesquisas epidemiológicas da asma, para indivíduos entre 20 e 44 anos de idade, têm utilizado o ECRHS, para investigar a população em geral e algumas categorias profissionais expostas a maior risco como os trabalhadores em minas de carvão, operários da construção civil ou agricultores.

Pode-se considerar que os funcionários de um hospital, independentemente da ocupação exercida, desempenham atividades ou prestam assistência direta e indireta ao paciente. Atuando sob supervisão de profissional de nível universitário, desempenham tarefas de complexidade variável, organizam o ambiente de trabalho e dão continuidade aos plantões. Essas atividades conferem melhor atendimento ao público externo, salvaguardando a imagem do hospital, maior tranqüilidade ao paciente, que pode acarretar maior expectativa de recuperação da saúde, e melhores condições de obediência aos padrões de qualidade. ${ }^{(13)}$ Assim sendo, seu adoecimento compromete a saúde dos pacientes, pois altera o equilíbrio da cadeia de processos envolvidos na prestação desses serviços.

Todos esses fatores são ainda mais importantes quando os funcionários trabalham em hospital público, universitário e de nível terciário, como é o caso do Hospital das Clínicas da Universidade Federal de Pernambuco, local da presente pesquisa. 
0 objetivo deste estudo é determinar a prevalência de asma brônquica em 351 funcionários do hospital-escola, com idade entre 20 e 44 anos, segundo os critérios do questionário do ECRHS. ${ }^{(14)}$

\section{MÉTODOS}

Por meio de um estudo descritivo de prevalência, aleatório e estratificado por setor hospitalar, foram analisados os dados de 351 dentre 651 funcionários (53,9\%), que obedeciam aos critérios de inclusão e exclusão.

Os critérios de inclusão foram: ser funcionário do hospital, estar no exercício de suas funções profissionais, ter idade entre 20 e 44 anos, e concordar em participar da pesquisa, após terem sido explicados os objetivos da mesma. Foi excluído um funcionário, devido à ausência de preenchimento de todos os sintomas do ECRHS, condição de validade das informações. ${ }^{(14)}$ Foram obedecidos os preceitos éticos determinados pela Resolução n ${ }^{\circ}$ 196/96 do Conselho Nacional de Saúde. ${ }^{(15)}$

0 Hospital das Clínicas da Universidade Federal de Pernambuco ocupa uma área de $64.614 \mathrm{~m} 2$, onde trabalham 1.578 funcionários, além de 306 docentes, 1.637 estudantes, dos quais 365 pós-graduandos, e 174 médicos residentes. Caracteriza-se por prestar assistência à saúde no nível terciário, dispondo de 213 ambulatórios, quinze hemodialisadores em unidade de Nefrologia, 416 leitos em enfermarias, dezoito em unidade de terapia intensiva neonatal e oito em unidade de terapia intensiva para adultos.

Em obediência aos critérios de validade adotados na tradução do ECRHS para outros idiomas, ${ }^{(14)}$ nesta primeira tradução para a língua portuguesa realizou-se a adaptação cultural local, por meio de aplicação do questionário a estudantes, funcionários do hospital e professores, devendo a validação ser realizada posteriormente.

As variáveis independentes constituíram-se em sintomas ou indícios, já que foram apenas informações prestadas pelos pesquisados, sem a constatação desses achados por parte do pesquisador. Foram elas: presença de ruídos adventícios (referência de barulho percebido pelo pesquisado ao respirar, como sibilos ou chiado); dispnéia com ruídos adventícios (referida como presença de sibilos ou chiado aos movimentos respiratórios, acompanhada de sensação de falta de ar); ruídos adventícios em ausência de gripe (informação do pesquisado de que sibilos ou chiados ocorreram nos doze meses anteriores à pesquisa, não concomitantes a episódio de gripe ou resfriado); sensação de aperto torácico (informação desse sintoma nos doze meses anteriores à pesquisa, independentemente de causa); dispnéia noturna (referência de o pesquisado ter acordado, espontaneamente, devido à falta de ar); tosse noturna (tosse que causou o despertar espontâneo do pesquisado, no período de doze meses anteriores à pesquisa); e uso de medicação (emprego de medicamentos, sob qualquer forma de apresentação farmacêutica, à época em que as informações foram prestadas, prescritos por médico, para o tratamento da asma).

Além das variáveis do questionário do ECRHS, foi considerado como variável independente o subtratamento como sendo o relato de crises de asma nos últimos doze meses, diagnosticadas por médico, em presença ou ausência de uso de medicação para asma.

Foi considerada como variável dependente a história de asma, caracterizada pela informação de crise asmática, diagnosticada por médico, nos doze meses que antecederam a pesquisa.

Os dados foram processados com o programa EPI-INFO 2002, revisão 1.0, do Centers for Disease Control and Prevention - Organização Mundial de Saúde. ${ }^{(16)} 0$ teste estatístico para análise da associação entre variáveis utilizado foi o qui-quadrado, realizado ao nível de significância de 0,05.

\section{RESULTADOS}

Foram estudados 351 funcionários com idade variando entre 21 e 44 anos (mediana de 37 anos), $63,3 \%$ deles com idade entre 35 e 44 anos. Considerada a distribuição por sexo, 99 eram do sexo masculino (28,2\%), com mediana etária de 36 anos, e 252 do feminino $(71,8 \%)$, com mediana etária de 37 anos.

A distribuição dos pesquisados segundo a escolaridade refletiu a atividade na área de saúde, tendo predominado o segundo grau completo, com 156 funcionários $(44,4 \%)$ e o terceiro grau, com 140 funcionários $(9,9 \%)$. Considerando-se as ocupações exercidas no hospital, 128 funcionários exerciam atividades que não exigiam contato com os pacientes $(38,0 \%)$, enquanto que 209 prestavam cuidados de saúde diretos, à beira do leito $(62,0 \%)$ ou nos ambulatórios (185 funcionários, 


\section{TABELA 1}

Distribuição de sintomas de asma investigados pelo ECRHS segundo o sexo de 351 funcionários do Hospital das Clínicas da Universidade Federal de Pernambuco, de abril a novembro de 2002

\begin{tabular}{|c|c|c|c|c|}
\hline \multirow{2}{*}{$\begin{array}{l}\text { Sintomas ou indícios de asma } \\
\text { investigados pelo ECRHS }\end{array}$} & \multicolumn{2}{|c|}{ Sexo $(\%)$} & \multirow[t]{2}{*}{ Total $(\%)$} & \multirow{2}{*}{$\begin{array}{c}\text { Teste } \\
\text { Estatístico }\end{array}$} \\
\hline & Feminino & Masculino & & \\
\hline Sibilos nos últimos 12 meses & $36(10,2)$ & $8(2,3)$ & $44(12,5)$ & $p=0,11$ \\
\hline Sibilos sem gripe & $30(8,5)$ & $8(2,3)$ & $38(10,8)$ & $p=0,30$ \\
\hline Dispnéia com sibilos & $29(8,3)$ & $9(2,6)$ & $38(10,8)$ & $p=0,13$ \\
\hline Dispnéia noturna & $66(18,8)$ & $15(4,3)$ & $83(23,6)$ & $p=0,03^{*}$ \\
\hline Sensação de aperto no peito & $38(10,8)$ & $7(2,0)$ & $45(12,8)$ & $p=0,04^{*}$ \\
\hline Tosse noturna & $76(21,6)$ & $15(4,3)$ & $91(25,9)$ & $p=0,001^{*}$ \\
\hline Uso de medicação para asma & $18(5,1)$ & $4(1,1)$ & $22(6,3)$ & $p=0,28$ \\
\hline
\end{tabular}

Percentuais calculados com base em 351 pacientes, já que cada um apresentava mais de um sintoma.

* dados estatisticamente significativos.

ECRHS: European Community Respiratory Health Survey.

$54,8 \%$ ), ou indiretos, por meio de serviços de higienização ambiental ou processamento de roupas (24 funcionários, 7,2\%).

Dos 351 funcionários avaliados, 326 forneceram a informação referente à existência de crise de asma nos doze meses anteriores à pesquisa. Trinta e cinco indivíduos relataram crise de asma nos doze meses anteriores à pesquisa $(10,7 \%)$, dos quais 29 eram do sexo feminino $(82,8 \%)$ e 6 do masculino $(17,2 \%)$. Embora tenha havido predomínio de episódio de asma entre mulheres, a diferença não foi estatisticamente significativa $(p=0,20)$.

Analisando-se a prevalência de crise de asma por faixa etária, detectou-se ter sido estatisticamente maior nos indivíduos com idade entre $20 \mathrm{e}$ 29 anos $(p=0,02)$.

A distribuição dos sintomas do ECRHS segundo o sexo dos 351 pacientes está expressa na Tabela 1. Identificou-se terem sido estatisticamente mais freqüentes no sexo feminino a dispnéia noturna $(p=0,03)$, a sensação de aperto no peito ( $p$ $=0,04)$ e a tosse noturna $(p=0,001)$.

Analisando-se a freqüência do relato dos sintomas relacionados à asma segundo o avançar da idade, detectou-se que, proporcionalmente, as faixas etárias não diferiram quanto à presença de sibilos nos últimos doze meses, sibilos com gripe, sensação de aperto no peito e tosse noturna, pois obedeceram à proporção amostral de 1:4 entre adultos jovens ( 20 a 29 anos) e adultos ( 30 a 44 anos). No entanto, os sintomas de dispnéia com sibilos e uso de medicação foram mais freqüentes entre os indivíduos com idade entre 20 e 29 anos, na proporção aproximada de 1:1 entre adultos jovens e adultos (Tabela 2).

Ordenando-se os sintomas ou indícios investigados no ECRHS, significativamente associados com crise de asma nos últimos doze meses, se-

TABELA 2

Distribuição de sintomas ou indícios de asma investigados pelo ECRHS segundo faixas etárias de 351 funcionários do Hospital das Clínicas da Universidade Federal de Pernambuco, de abril a novembro de 2002

\begin{tabular}{lcccc}
\hline Sintomas ou indícios de asma & \multicolumn{2}{c}{ Faixas etárias $(\%)$} & Total $(\%)$ & $\begin{array}{c}\text { Teste } \\
\text { investigados pelo ECRHS }\end{array}$ \\
\hline Sibilos nos últimos 12 meses & $20-29$ & $30-44$ & $44(12,5)$ & $\mathrm{p}=0,41$ \\
Sibilos sem gripe & $10(2,8)$ & $34(9,7)$ & $38(10,8)$ & $\mathrm{p}=0,36$ \\
Dispnéia com sibilos & $9(2,6)$ & $29(8,3)$ & $35(10,0)$ & $\mathrm{p}=0,01^{*}$ \\
Dispnéia noturna & $12(3,4)$ & $23(6,6)$ & $81(23,1)$ & $\mathrm{p}=0,09$ \\
Sensação de aperto no peito & $20(5,7)$ & $61(17,4)$ & $45(12,8)$ & $\mathrm{p}=0,74$ \\
Tosse noturna & $9(2,6)$ & $36(10,2)$ & $90(25,6)$ & $\mathrm{p}=0,85$ \\
Uso de medicação para asma & $17(4,8)$ & $73(20,8)$ & $22(6,3)$ & $\mathrm{p}=0,01^{*}$ \\
\hline
\end{tabular}

Percentuais calculados com base em 351 pacientes, já que cada um apresentava mais de um sintoma.

* dados estatisticamente significativos.

ECRHS: European Community Respiratory Health Survey. 


\section{TABELA 3}

Distribuição dos sintomas ou indícios de asma investigados pelo ECRHS e crise de asma nos últimos doze meses, referida por 35 funcionários do Hospital das Clínicas da Universidade Federal de Pernambuco, de abril a novembro de 2002

\begin{tabular}{lcc}
\hline Sintomas ou indícios de asma & \multicolumn{3}{c}{$\begin{array}{c}\text { Relato de crise de asma } \\
\text { investigados pelo ECRHS }\end{array}$} & $\begin{array}{cc}\text { nos últimos 12 } \\
\text { no }\end{array}$ & de pacientes & $\%$ \\
\hline Sibilos nos últimos 12 meses & 28 & 80,0 \\
Dispnéia com sibilos & 26 & 74,2 \\
Sibilos sem gripe & 25 & 71,4 \\
Tosse noturna & 23 & 65,7 \\
Dispnéia noturna & 21 & 60,0 \\
Uso de medicação para asma & 20 & 57,1 \\
Sensação de aperto no peito & 18 & 51,4 \\
\hline
\end{tabular}

Percentuais calculados com base em 351 pacientes, já que cada um apresentava mais de um sintoma.

ECRHS: European Community Respiratory Health Survey.

gundo a freqüência, observaram-se: sibilos nos últimos doze meses, dispnéia com sibilos, sibilos sem gripe, tosse noturna, dispnéia noturna, uso de medicação para asma e sensação de aperto no peito (Tabela 3).

Dentre os 326 funcionários que responderam ao item crise de asma nos últimos doze meses, 21 informaram estar em uso de medicação para asma à época da pesquisa $(6,4 \%)$, independentemente de prescrição médica, dos quais 20 relataram crise de asma diagnosticada por médico $(95,2 \%)$ e um negou-a $(4,8 \%)$. Dentre os 35 funcionários que declararam crise de asma no ano anterior à pesquisa, 20 estavam em tratamento $(57,1 \%)$ e 15 negaram-no $(42,9 \%)$, e constituíram-se em casos de subtratamento, por insuficiência de dose e inadequação na escolha do medicamento ou ausência de terapêutica medicamentosa, respectivamente (Tabela 4).

\section{DISCUSSÃO}

No presente trabalho, a prevalência dos sintomas de asma foi superior à observada no Canadá, ${ }^{(4)}$ na zona rural de Beijing (China), ${ }^{(17)}$ na Antuérpia (Bélgica) ${ }^{(18)}$ e na Espanha, ${ }^{(19)}$ talvez porque todos esses estudos tenham tido base populacional e tenham sido realizados em locais com características climáticas e ambientais bastante distintas daquela da cidade do Recife (PE), ou, ainda, devido à diferença das características socioeconômicas entre os indivíduos desses estudos e os funcionários do Hospital das Clínicas da Universidade Federal de Pernambuco.

Há autores que consideram como uma das hipóteses mais interessantes, que requer novas pesquisas, a potencial contribuição do meio ambiente e dos fatores genéticos para essas diferenças. ${ }^{(20)}$ Outros julgam que a atuação de fatores genéticos parece pouco provável, já que estudos epidemiológicos de prevalência, repetidos em vários países, mostraram alterações significativas em curto espaço de tempo, incompatíveis com causas genéticas, de ação lenta em populações. ${ }^{(19)}$

0 ECRHS ${ }^{(14)}$ e o International Union Against Tuberculosis and Lung Disease foram comparados quanto à eficiência em detectar asma leve. ${ }^{(21)}$ Para tanto, foram estudados os dados de 196 pacientes com idade entre 16 e 59 anos, com queixas pulmonares ou extrapulmonares, nunca antes examinados para asma brônquica. Subdivididos em dois grupos, na primeira fase responderam ao ECRHS ou ao International Union Against Tuberculosis and Lung Disease. Aqueles que referiram pelo menos um sintoma em qualquer dos questionários foram submetidos a exames clínicos, funcionais e alergológicos, fornecendo resultados que permitiram aos autores concluir que o ECRHS deve ser o

TABELA 4

Distribuição do uso de medicação para asma de 326 funcionários, com história de asma nos últimos doze meses, do Hospital das Clínicas da Universidade Federal de Pernambuco, de abril a novembro de 2002

\begin{tabular}{|c|c|c|c|c|c|}
\hline \multirow[t]{2}{*}{ Uso de medicação para asma } & Relato & Relato de crise de asma nos últimos 12 meses diagnosticada por médico & $2 \mathrm{me}$ & gnostic & total \\
\hline & $\mathrm{n}$ & $\%$ & $\mathrm{n}$ & $\%$ & \\
\hline sim & 20 & 57,1 & 1 & 0,4 & 21 \\
\hline não & 15 & 42,9 & 290 & 99,6 & 305 \\
\hline Total & 35 & 100,0 & 291 & 100,0 & 326 \\
\hline
\end{tabular}

25 funcionários omitiram a informação referente a crise de asma nos últimos doze meses.

Percentuais calculados com base nos totais por categoria. 
questionário de escolha para levantamentos epidemiológicos sobre asma. Por esse motivo, ele foi adotado como instrumento de coleta na presente pesquisa (trata-se de questionário internacionalmente validado). ${ }^{(22)}$

No ECRHS, a asma é conceituada como a referência a crise asmática nos últimos doze meses ou uso de medicação para seu tratamento. No entanto, por se reconhecer a possibilidade de viés de memória por parte dos entrevistados, de erro de classificação de um episódio de dispnéia como asma feita pelo entrevistado sem diagnóstico médico, e da automedicação, procedimento freqüente em nosso meio, no presente trabalho considerou-se asma exclusivamente a informação de crise nos doze meses anteriores à pesquisa. Restringiu-se assim o conceito do ECRHS, tal como adotado por outros autores, ao investigarem o aumento da prevalência de asma em três cidades italianas, comparando o período de 1998 a 2000 ao de 1991 a $1993 .{ }^{(23)}$

0 presente estudo foi de fase 1 , em área restrita a um hospital universitário, como forma de levantamento epidemiológico pioneiro, para que se pudessem obter dados iniciais que caracterizassem a prevalência de crises de asma nos últimos doze meses entre seus funcionários.

A análise dos subtratamentos também foi contemplada, porque era de se supor que esse evento estivesse ausente ou em freqüência reduzida, pelo fato desses funcionários terem um nível diferenciado de educação para a saúde em relação à população em geral, pelo convívio diário com as enfermidades, o que os levaria a buscar tratamento com maior freqüência.

Outro aspecto da metodologia adotada no presente trabalho refere-se à coleta dos dados por meio de questionário de auto-resposta, que, comprovadamente, não é considerada como a melhor opção para se pesquisar asma.

Foi desenvolvido um estudo, classificado por seus autores como inovador, pragmático, aleatório e controlado, para avaliação da sensibilidade, da aceitação e da eficiência dos cuidados primários a pacientes asmáticos mediante consulta telefônica. ${ }^{(24)}$ Nas conclusões, comprovaram que tais consultas possibilitam assistência a $26 \%$ mais pacientes que as ambulatoriais ou os questionários de auto-resposta, sem qualquer desvantagem clínica, sem perda de satisfação e com maior liberdade do entrevistado em expressar seus sintomas, suas dúvidas e a eventual evasão do tratamento. Esses autores afirmaram que as pesquisas sobre sintomas de asma deveriam ser feitas preferencialmente por telefone. Embora essa metodologia tenha sido comprovada e validada também pelos levantamentos epidemiológicos populacionais do ECRHS, não pode ser aplicada no presente trabalho, dada a baixa condição socioeconômica dos funcionários do hospital que exerciam ocupações administrativas ou de serviços de limpeza e higiene (nem todos tinham telefone em casa), o que representaria uma perda significativa no tamanho amostral máximo, da ordem de 39\%.

Embora o presente trabalho não tenha fixado como objetivo uma análise referente à asma ocupacional, não se pode ignorar o alerta de Samet ${ }^{(20)}$ quanto à influência do meio ambiente de locais restritos sobre a prevalência de asma, o que poderia explicar alguns dos resultados do presente estudo, pois o ambiente hospitalar pode atuar como fator de risco para sintomas de asma.

Neste estudo, a crise de asma foi mais freqüente em mulheres, o que pode ser explicado pelos resultados de outros autores, que identificaram que as mulheres têm responsividade brônquica maior que a dos homens, sendo quase totalmente devida a seu menor calibre de vias aéreas. ${ }^{(25)}$ Essa mesma afirmação foi feita por outros autores que, ao estudarem 18.659 pessoas, sendo 52,1\% mulheres e $47,9 \%$ homens, residentes em dezesseis países, mediante a aplicação do ECRHS, identificaram que a incidência de asma na faixa etária de zero a 44 anos mantém uma relação inversa com a idade e predomina em mulheres. ${ }^{(26)}$

Também foram estatisticamente significativas as maiores prevalências de dispnéia noturna, sensação de aperto no peito e tosse noturna entre as mulheres, para as quais não se encontrou referência na literatura consultada. Esses dados podem estar relacionados ao exercício de ocupações em ambientes menos seguros e de maior insalubridade, como os de cozinha, processamento de roupas, limpeza e higienização ambiental, onde elas são submetidas a alterações bruscas de temperatura (quando da retirada de alimentos da câmara frigorífica) e a vapores (quando do preparo dos alimentos e no ato de passar roupas).

0 predomínio de referência de asma em indivíduos mais jovens concordou com a história natural da doença, mas discordou do estudo de ou- 
tros autores que analisaram a prevalência de asma por idade. ${ }^{(27)}$ Uma possibilidade para os resultados da presente pesquisa são as características inerentes ao trabalho em hospital, que submete os indivíduos a níveis de estresse e de poluição muito altos, exigindo-lhes intensas adaptações para que possam conviver com o sofrimento alheio e manter sua estabilidade psíquica. Essas adaptações desgastam mais os indivíduos de 20 a 29 anos de idade, provavelmente pelo menor tempo de aprendizado da adaptação a situações conflitantes e de sofrimento.

Pode-se aventar a hipótese de que, nessa população, os fatores pessoais, comportamentais e profissionais possam estar atuando em ambos os sexos, hipótese que se respalda em outro trabalho, quanto ao componente neurotizante da asma sobre os pacientes. ${ }^{(28)}$ Nesse estudo, que analisou 426 adultos participantes da fase 11 do ECRHS, identificou-se que a presença de sibilos nos últimos doze meses afetava gravemente a qualidade de vida dos indivíduos, muito mais que episódios de asma ou hiper-responsividade brônquica isolada, embora esses dois sinais piorassem muito o estado mental dos portadores.

0 achado de 12,5\% de indivíduos com sibilos nos últimos doze meses na amostra ora estudada foi importante, pois indicou comprometimento na qualidade de vida dos funcionários do hospital e, indiretamente, na dos pacientes por eles atendidos. ${ }^{(29)}$

A identificação de 35 funcionários de um hospital universitário com provável subtratamento para a asma remete à reflexão da prevenção e da redução da morbidade da asma, que pode ser obtida através de intervenções educacionais.

Em um estudo no Canadá( ${ }^{(30)}$ e em outro no Bra$\mathrm{sil}^{(9)}$, após doze meses de vivência de um programa educacional para asma, obteve-se significativa melhora no conhecimento da doença, na disposição para ajustar a medicação, no escore de qualidade de vida e no pico de fluxo expiratório. No entanto, nenhum programa de educação, assim como nenhum aprimoramento do tratamento de asma, é válido se não houver forte adesão dos pacientes às orientações propostas, associada ao entendimento da doença, com conseqüente melhora na aceitação terapêutica.

No campo da asma, as investigações epidemiológicas não devem estar limitadas a grandes populações urbanas de diferentes países ou continentes, visto que o estudo em áreas relativamente restritas pode fornecer informações que venham a elucidar diferentes causas de sua prevalência, tal como ocorreu no presente trabalho, que sugeriu a necessidade de novos levantamentos, mais detalhados, que possam contribuir para o bem-estar dos pacientes asmáticos e qualidade dos serviços hospitalares.

\section{REFERÊNCIAS}

1. Burney PG, Chinn S, Rona R. Has the prevalence of asthma increased in children? Evidence from the national study of health and growth 1973-1986. BMJ. 1990;300(6735):1306110.

2. Weiss KB, Wagener DK. Changing patterns of asthma mortality. Identifying target populations at high risk. JAMA. 1990;264(13):1683-7.

3. Reid DD, Abramson M, Raven J, Walters HE. Management and treatment perceptions among young adults with asthma in Melbourne: the Australian experience from the European Community Respiratory Health Survey. Respirology. 2000;5(3):281-7.

4. Manfreda J, Becklake MR, Sears MR, Chan-Yeung M, DimichWard $\mathrm{H}$, Siersted HC, et al. Prevalence of asthma symptoms among adults aged 20-44 years in Canada. CMAJ. 2001;164(7):995-1001.

5. Godoy DV, Dal Zotto C, Bellicanta J, Weschenfelder RF, Nacif SB. Doenças respiratórias como causa de internações hospitalares de pacientes do Sistema Único de Saúde num serviço terciário de clínica médica na região nordeste do Rio Grande do Sul. J Pneumol. 2001;27(4):193-8.

6. Brasil. Ministério do Trabalho e Emprego. CBOClassificação Brasileira de 0cupação. [texto na Internet].Versão 1.0 reduzida. 2002. [citada 2003 Maio 10].Brasília: Ministério do Trabalho e Emprego. Disponível em: http://www.mtecbo.gov.br/index.htm

7. Menezes AMB. Epidemiologia. In: Correa SLC. Condutas em pneumologia. Rio de Janeiro: Revinter; 2001. p.42-9.

8. Sociedade Brasileira de Alergia e Imunologia. Sociedade Brasileira de Pediatria e Sociedade Brasileira de Pneumologia e Tisiologia. 111 Consenso Brasileiro no Manejo da Asma. Capítulo 1. Definiçäo, epidemiologia, patologia e patogenia. J Pneumol. 2002;28(Supl 1):S4-S5.

9. Oliveira MA, Muniz MT, Santos LA, Faresin SM, Fernandes ALG. Custo-efetividade de programa de educação para adultos asmáticos atendidos em hospital-escola de instituição pública. J Pneumol. 2002;28(2):71-6.

10. Sears MR. Changing patterns in asthma morbidity. J Investig Allergol Clin Immunol. 1995;5(2):66-72.

11. Evans D. To help patients control asthma the clinician must be a good listener and teacher. Thorax. 1993;48(7):684-7.

12. Vieira JE, Cukier A, Stelmach R, Kasahara D1, Gannam S, Warth M. Comparison of knowledge on asthma: doctors completing internal medicine residence and doctors completing medical school. São Paulo Med J. $2001 ; 119(3): 101-4$.

13. Brasil. Ministério da Saúde. Conselho Nacional de Saúde. Resolução 196/96 do Conselho Nacional de Saúde. Diretrizes e normas regulamentadoras de pesquisas envolvendo seres humanos [texto na Internet]. [citado 
2002 Jan 2]. Disponível em: http://www.unifesp.br/ reitoria/ orgaos/comites/etica/resolucao6.htm

14. European Community Respiratory Health Survey (ECRHS). Variations in the prevalence of respiratory symptoms, selfreported asthma attacks, and use of asthma medication in the European Community Respiratory Health Survey (ECRHS). Eur Respir J. 1996;9(4):687-95.

15. Brasil. Ministério da Saúde - DATASUS. Morbidade hospitalar por local de residência - Pernambuco - asma - 20 a 44 anos, jan/1996-jan/2003 [texto na Internet]. [citado 2003 Mar 19]. Disponível em http>//tabnet.datasus.gov.br/cgi/ tabcgi.exe?sih/cnv/mespe.def.

16. Centers for Disease Control and Prevention - CDC. Vital and health statistics: current estimates from the National Health Interview Survey, 1996. Atlanta US: Department of Health and Human Services; 1999.

17. Chan-Yeung M, Zhang LX, Tu DH, Li B, He GX, Kauppinen R, et al. The prevalence of asthma and asthma-like symptoms among adults in rural Beijing, China. Eur Respir J. 2002;19(5):853-8.

18. Wieringa MH, Weyler JJ, Nelen VJ, Van Hoof KJ, Van Bastelaer F, Van Sprudel MP, et al. Prevalence of respiratory symptoms: marked differences within a small geographical area. Int J Epidemiol. 1998;27(4):630-5.

19. Upton MN, McConnachie A, McSharry C, Hart CL, Smith GD, Gillis $C R$, et al. Intergenerational 20 year trends in the prevalence of asthma and hay fever in adults: the Midspan family study surveys of parents and offspring. BMJ. 2000;321(7253):88-92.

20. Samet JM. A historical and epidemiological perspective on respiratory symptoms questionnaires. Am J Epidemiol. 1978:108(6):435-46.

21. Sergeeva GR, Emel'ianov AV, Ivanova N1. Effectiveness of detection of bronchial asthma with two internationa questionnaires. Ter Arkh. 2002;74(3):12-4. Russian.
22. Samet JM. Asthma Epidemiology: old and new issues. In: Neffen HE, Baena-Cagnani CE, Fabbri L, Holgate S, O'byrne P. Asthma - A link between environment, immunology, and the airways. Procedings of the XVlth World Congress of Asthma. Argentina : Hogrefe \& Huber; 1999. p.27-32.

23. Verlato G, Corsico A, Villani S, Cerveri l, Migliore E, Accordini $\mathrm{S}$, et al. Is the prevalence of adult asthma and allergic rhinitis still increasing? Results of an italian study. J Allergy Clin Immunol. 2003;111(6):1232-8.

24. Pinnock H, Bawden R, Proctor S, Wolfe S, Scullion J Price D, et al. Accessibility, acceptability, and effectiveness in primary care of routine telephone review of asthma: pragmatic, randomised controlled trial. BMJ. 2003;326(7387):477-9.

25. Schaubel DH, Johansen H, Mao Y, Dutta M, Manfreda J. Risk of preschool asthma: incidence, hospitalization, recurrence, and readmission probability. J Asthma. 1996;33(2):97-103.

26. De Marco R, Zanolin ME, Accordini S, Signorelli D, Marinoni A, Bugiani M, et al. A new questionnarie for the repeat of the first stage of the European Community Respiratory Health Survey: a pilot study. Eur Respir J. 1999;14(5):1044-8.

27. Campello C, Ferrari M, Poli A, Olivieri M, Tardivo S, Verlato $\mathrm{G}$, et al. Prevalence of asthma and asthma-like symptoms in an adult population sample from Verona. ECRHS Verona. European Community Respiratory Health Survey. Monaldi. Arch Chest Dis. 1998;53(5):505-9.

28. Matheson M, Raven J, Woods RK, Thien F, Walters EH, Abramson M. Wheeze not current asthma affects quality of life in young adults with asthma. Thorax. 2002;57(2):165-7.

29. Huovinen E, Kaprio J, Koskenvuo M. Asthma in relation to personality traits, life satisfaction, and stress: a prospective study among 11,000 adults. Allergy. 2001;56(10):971-7.

30. Coté J, Bowie D, Robichaud P, Parent J, Battisti L, Boulet L. Evaluation of two different educational interventions for adult patients consulting with an acute asthma exacerbation. Am J Respir Crit Care. 2001;163(6):1415-9. 